\title{
Rapid Microvalve Actuated Electroosmotic Reagent Delivery to Surface-Immobilized Molecules
}

\author{
Hao Chen* \\ University of Michigan, USA \\ *Corresponding author: Hao Chen, University of Michigan, Biomedical Engineering, Ann Arbor, MI. 48109, USA \\ Submission: November 17, 2017; Published: January 10, 2018
}

\begin{abstract}
We present a micro fluidic device that allows rapid and defined delivery of discrete and homogeneous reagents or samples to allow kinetic studies of surface-tethered biomolecules. We developed an Electro Osmotic Flow (EOF) based device consisting of an asymmetric Y-junction with an incorporated fast, pressure driven valve, two embedded measurement electrodes and reservoirs. The EOF is used to circumvent kinetic limitations on reagent transport to the surface of these tethered biomolecules due to the slow diffusion across parabolic concentration gradients in conventional pressure-driven flow devices. Using Fluorescein isothiocyanate (FITC) as the $\mathrm{pH}$ sensitive surface-immobilized biomolecules, we show that the reagent solution can be repeatedly exchanged within milliseconds, and that by using a synchronized triggering scheme we can monitor the reaction of our sensor biomolecules to the change of the environment on a time scale of $10 \mathrm{~ms}$.
\end{abstract}

\section{Introduction}

Micro fluidic devices and microarray technology have become important tools for modern biochemical and biomedical analysis for their quick and effective testing of micro size samples with exceedingly high sensitivity [1]. The reduction in reaction volume in these types of assays is accompanied by an increasing importance of designs accounting for micro-scale volumetric and physiological effects [2] at the fluid/solid or fluid/gas interface, fluidic properties in the nano-liter regime, modified surface-targeted techniques with shorter reaction times, and variable boundary conditions that lead to significant changes in flow characteristics. The latter effect is exemplified by the fact that all flows become laminar at typical micro fluidic dimensions and velocities, resulting in a parabolic velocity profile of pressure-driven flows (PDF) across micro channels. This makes the delivery of samples to molecules that are anchored at the channel walls difficult, as diffusion across the parabolic concentration gradient to the walls is comparatively slow. This effect limits those experiments that require the study of folding and unfolding kinetics of single or low number of biomolecules that undergo fast responses to changes in the chemical environment near a wall. In contrast to inorganic chemical reactions, the activity of biomolecules such as proteins, DNA [3], and especially RNA and aptameric enzymes [4], relies primarily on the spatial configuration of their active sites. It has also become apparent that misfolding or insufficient folding of proteins cause intra-cellular stress responses [5] and may be associated with a pathway to neurodegenerative diseases [6]. Due to the complex energy landscape of typical folding and unfolding processes, we are presented with many possible pathways to the final functional state or states, and it is therefore essential that a reliable system be used to unambiguously identify these intermediary states $[7,8]$. Due to these constraints, single-molecule approaches seem to be more effective than bulk measurements, although these approaches also have their limitations. For example, photochemical triggering studies require secondary attached motifs that result in a limited folding landscape or lower folding entropy [9-15], ultrafast mixing studies require channel dimensions on the order of $50 \mathrm{~nm}$ that would jeopardize folding due to its closeness or even smaller dimensions compared to the inherent radius of gyration of many biomolecules of interest, not mentioning the user-unfriendly clogging [16], highly questionable reusability [17], and image variability due to pressure fluctuations [18]. The buffer exchange times for pressure driven flow based systems are ten times slower compared to those of EOFs [19], and are therefore mostly suitable for the study of larger proteins with longer folding response times such as those of rotary bimotors' inactivation rates [20]. Although there some studies that show the use of nanoliter volumes to electro kinetically controlled DNA hybridization, the fluid of interest that is pushed and sandwiched between the two neutral buffers would become diluted due to diffusion at the interphases with these buffers [21], therefore defeating the purpose of producing discrete and homogeneous environmental changes.

Our proposed design of a micro fluidic device to perform single molecule kinetic studies of surface-tethered biomolecules alleviates many of these problems. Our device permits complete 
buffer exchanges at the surface on timescales of milliseconds by replacing pressure-driven flow with mechanically switched EOF via a thoroughly separated PDMS based valve. The molecules of interest are tethered on glass located at the bottom of the channel, and total internal reflection (TIRF) is used to focus on those molecules tethered on glass only. The reaction is observed by fluorescence microscopy at the junction of three channels carrying the different solutions of interest towards the tethered molecules. Switching between the solutions in the reaction surface area is accomplished by rapidly opening and closing a pneumatically controlled valve that eliminate the diffusion between buffers. For example, when the main channel's valve is closed, the solution in the channel immediately to the left of the valve can be maintained at a certain $\mathrm{pH}$, while the solution immediately to the right of the valve can be exchanged or left to vary without altering the contents to the left of the valve.EOF is continuously on and is used to drive the solution of interest through the main channel that becomes more active when the valve is opened. This results in a steeper concentration gradient and thinner diffusion layer near the surface when compared to PDF, leading to a fast, efficient and reproducible reagent delivery mechanism that can be switched on within 10 milliseconds.

\section{Methods}

A modified $Y$ junction with channel cross sections of 110 by $8.5 \mu \mathrm{m}^{2}$ was manufactured by spin-coating PDMS (4:1 resin to catalyst, RTV615 kit, GE Silicones, Wilton, CT) onto a lithographically patterned wafer at $1900 \mathrm{rpm}$ for $50 \mathrm{~s}$, resulting in a total layer thickness of $35 \mu \mathrm{m}$. A second, top layer (10:1) containing the pneumatic valves ( 120 by $9 \mu \mathrm{m}^{2}$ ) was similarly patterned, carefully positioned on top of the first layer, and allowed to anneal. The PDMS chip containing both layers was then plasma bonded onto a glass cover slip that was patterned with $\mathrm{Au}$-Ti electrodes $(\mathrm{hTi}=5 \mathrm{~nm}$, $\mathrm{hAu}=55 \mathrm{~nm}$ ) using a conventional high vacuum evaporation coater. In order to protect the chip, the fluidic reservoirs, and the electrode interconnects from possible damages during handling and mounting due to external forces, the chip was embedded in an aluminum frame and protected using epoxy resin. The final design and implementation of this chip is shown in (Figure 1) and more thorough descriptions of this platform can be found in our earlier publications $[22,23]$. The glass bottom surfaces of the micro channels were silanized by injecting a 1:49 (v/v) mixture of 3-amino-propyltriethoxysilane (APTES, Sigma, St. Louis, MO) and acetone into the flow channels and incubating them for a maximum of 20 s to avoid damage to the valves. After drying the channels with nitrogen gas, pH-sensitive fluorescine-5-isothicyanate (FITC, $1 \mathrm{mg} / \mathrm{ml}$ in DMSO) molecules were allowed to attach to the aminemoiety of APTES on the channel surfaces for at most $8 \mathrm{~h}$ at $4{ }^{\circ} \mathrm{C}$ since longer incubations resulted in the formation of residue and PDMS degeneration impeding proper fluid actuations. Fluorescence images of the microchip were collected via a home-built, TIRF microscope using a 60x objective (Olympus, Plan-Apo, NA=1.45) with a cooled CCD camera (Cascade, Photometrics, Tucson, AZ). Sample illumination using an Argon-Ion laser (532nm) was controlled using a fast switching acousto-optic modulator (AOM, Gooch \& Housego, Bedford, MA). Image acquisition, laser pulse triggering, valve opening, and EOF actuation were triggered and timed using a custom-built analogue electronic synchronization system (ESS). The acquired data was first processed and then saved via Lab view (Austin, TX). The study system comprised of the PDMS micro fluidics chip with embedded electrodes, fluidic manifold, controllers, modulators, TIRF microscope, optical setup, and peripheral components are shown in (Figure 2). The PDMS chip is embedded in the aluminum block via epoxy, placed above the inverted TIRF Microscope's platform, buffer external lines connected onto the control valves, and the main channel are plugged into the pressure manifold. The electronic synchronization system is Lab view coded to modulate the AOM laser modulator, CCD camera trigger, control valves, electrode voltages, manifold pressure valves, buffer line inflow, signal the high voltage supply to activate EOF, and provide "valve open/close" feedback from a conductivity sensor across the main channel.

\section{Results and Discussion}

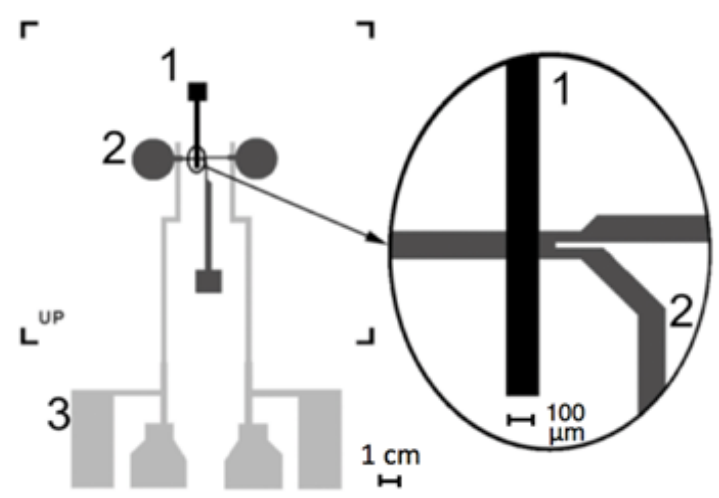

Figure 1: Layout of a microfluidic chip on a Au-Ti patterned cover glass. The picture shows the main valve channel.

a. With Y shaped fluidic channel

b. Beneath the valve (see inset). Two electrodes

c. With pads for wire bonding are coated onto the cover glass. 


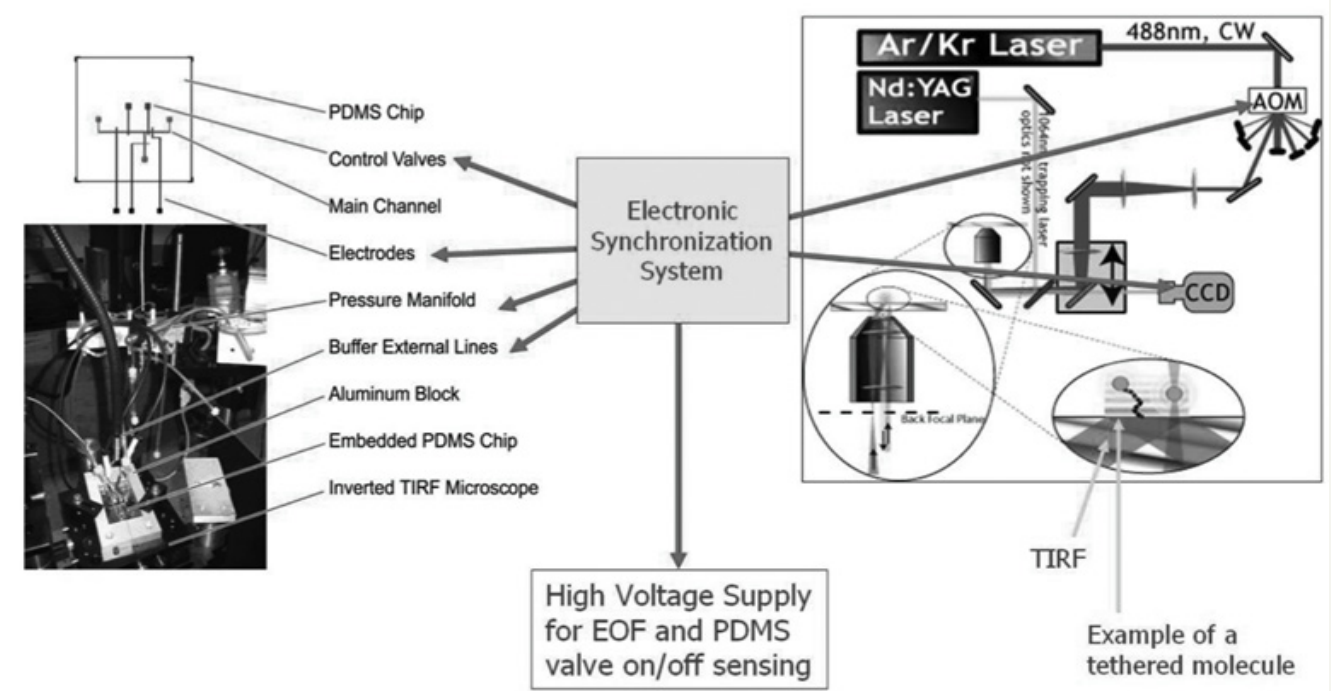

Figure 2: The study of the microfluidic chips required the synchronization of different electronic controllers, reagent delivery manifolds, and optical components.

An effective reagent exchange system allows for effective switching between discrete and highly homogeneous solutions in the surface area with tethered molecules of interest, leading way to a reproducible reagent delivery mechanism that can be switched on and off without mechanically disturbing the environment. To allow for the delivery of the compartmentalized fluid of interest, we optimized the dimensions and shape for the valve, fluidic channels, and the valve driving pressures [22] to allow the valves to open within milliseconds using conventional PDMS-based microfluidic components $[22,23]$. To decrease the diffusion distance between the molecules and solution of interest, EOF was used to deliver plug-shaped flows to produce steep concentration gradients near the surface of interest that contrasts to a much longer diffusion time using parabolic Hagen-Poiseuille flows. To demonstrate the capability of this delivery system, the prepared microfluidic chip with the surface-attached FITC as fluorescent $\mathrm{pH}$ sensor was secured on the inverted microscope stage described in the previous section. The device was setup by first priming the control valves were with deionized water to prevent bubble formation and attaching the pressure and reagent delivery manifold to the inlets of the chip, followed by checking the valve and main channel integrity using a short testing routine. The electrode contacts were then attached to the Electronic Synchronization System (ESS) provided the high voltages needed for the EOF and controlled the triggering scheme. The ESS system functioned as a hub to channel data and to quickly trigger image acquisition, laser pulse triggering, valve opening, and EOF actuation via a main trigger via a custom Lab view code that operated in a single shot or cyclic mode. Thus, all time-critical operations were hardware-implemented in the ESS to avoid introducing jitter from unpredictable delays introduced by the computer, operating system, and Lab view software.

We employed micro particle image-based velocimetry to characterize the mean flow velocities at different Electroosmotic driving voltages for each chip. The velocity profile $\mathrm{u}(\mathrm{x})$ as a function of the electric potential $\mathrm{E}$ for $10 \mathrm{mM} \mathrm{NaBr}$ at $\mathrm{pH} 9.1$ followed the theoretical expectation:

$$
u(x)=\left(1-\frac{\cosh \left(\frac{x}{\lambda_{D}}\right)}{\cosh \left(\frac{h}{2 \lambda_{D}}\right)}\right) v_{e o} \quad v_{e o}=\frac{\zeta \varepsilon E}{\eta}
$$

Which is derived from the PDF and gravity-free Navier Stokes equation in the low Zeta-potential limit [24]? Our observed values near the center of the channel, where dominant $\mathrm{x}$ is much larger than the Debye length, $\lambda D$, allowed for more homogenous data collection and are shown in (Figure 3). In this figure, the electrode separation for this particular chip was $19 \mathrm{~mm}$, the normalized Zeta Potential $\zeta / \mathrm{pC}$ at the wall was approximately $31 \mathrm{Mv}$ [25-27], the relative dielectric constant for water used was $\varepsilon r=80$, the viscosity $\eta$ of water used at $25^{\circ} \mathrm{C}$ was $0.89 \mathrm{mPas}$, and the voltages used to drive the EOF were limited to $800 \mathrm{~V}$. We noted that higher voltages led to random bubble formations in the channels due to redox reactions on the integrated and etched Ti-Au electrodes. The timing sequence for buffer exchange and data acquisition is shown in (Figure 4) and this triggering scheme primarily consists of driving the voltage for the EOF, opening a relief valve in the manifold to actuate the microfluidic valve, opening the camera exposure, and firing a laser pulse for stroboscopic sample illumination. The primary cycle trigger is directly routed to the EOF power supply to build Electroosmotic pressure across the valve during the closed state, and during the opened state that is actuated after a delay of $\mathrm{t} 1$ which is optimized independently for each chip. The definition of this delay $\mathrm{t} 1$ is mostly defined by the time the pneumatic system took to reduce the air pressure to the point where the different microfluidic valves opened, and thus varied slightly from chip to chip. Similarly, the camera exposure cycle delay $\mathrm{t} 2$ is mostly defined by the time to ready the camera before the valve physically opened; this delay however should not be set too long as unnecessarily exposure 
time usually increases camera noise. Typical camera exposure time signals started $10 \mathrm{~ms}$ before the valve opening signals. Since there was considerable timing jitter in the physical opening of the microfluidic valves, we used the resistance drop across the valve that denoted real time valve opening as a secondary trigger delay to start the laser pulse. Two $\mathrm{Au}-\mathrm{Ti}$ electrodes $(\mathrm{hTi}=5 \mathrm{~nm}, \mathrm{hAu}=55 \mathrm{~nm})$ were patterned on either side of the valve to measure the resistance drop across during valve-actuated buffer exchanges. To avoid accidental misfiring of the laser pulse, the secondary trigger was armed only after the real time valve actuation was sensed. The delay after the real time valve opening $t R$, and the length of the laser pulse $t L$, were both programmed to trigger within $1.5 \mu \mathrm{s}$. After the main valve closed $(\mathrm{tB}=1 \mathrm{~s})$, the measurement cycle was concluded by opening an external buffer valve and flushing the sample area for several seconds with buffer solution at $\mathrm{pH} 7.4$, at which point the sample delivery cycle could be triggered and analyzed again. Theoretically, when EOF is initiated, the diffusive layer created by a typical PDF is predicted to be larger than $400 \mathrm{nmat}$ approximately $37 \mu \mathrm{m}$ from the valve's edge, while an EOF profile can potentially be tuned to be smaller than $100 \mathrm{~nm}$, which is within the TIRF evanescent wave (1/e) distance of $120 \mathrm{~nm}$, thus allowing exponentially more accurate fluorescent signal to track the dynamics of tethered molecules via TIRF microscopy.

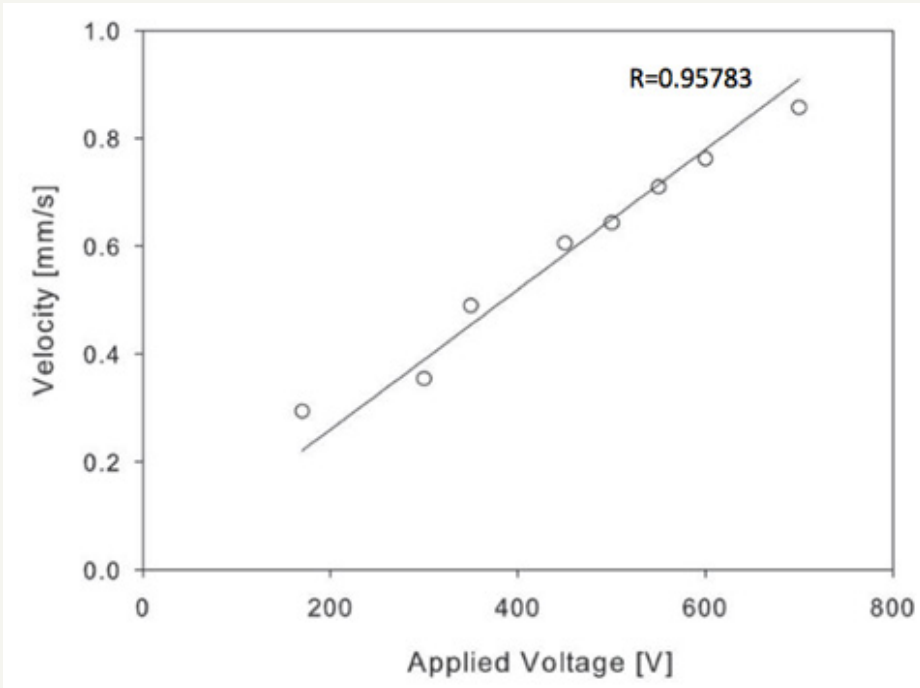

Figure 3: Velocity of the EOF flow as a function of applied voltage. Above a voltage of $800 \mathrm{~V}$ the flow became disturbed by the formation of bubbles due to a redox reaction at the electrodes on the chip.

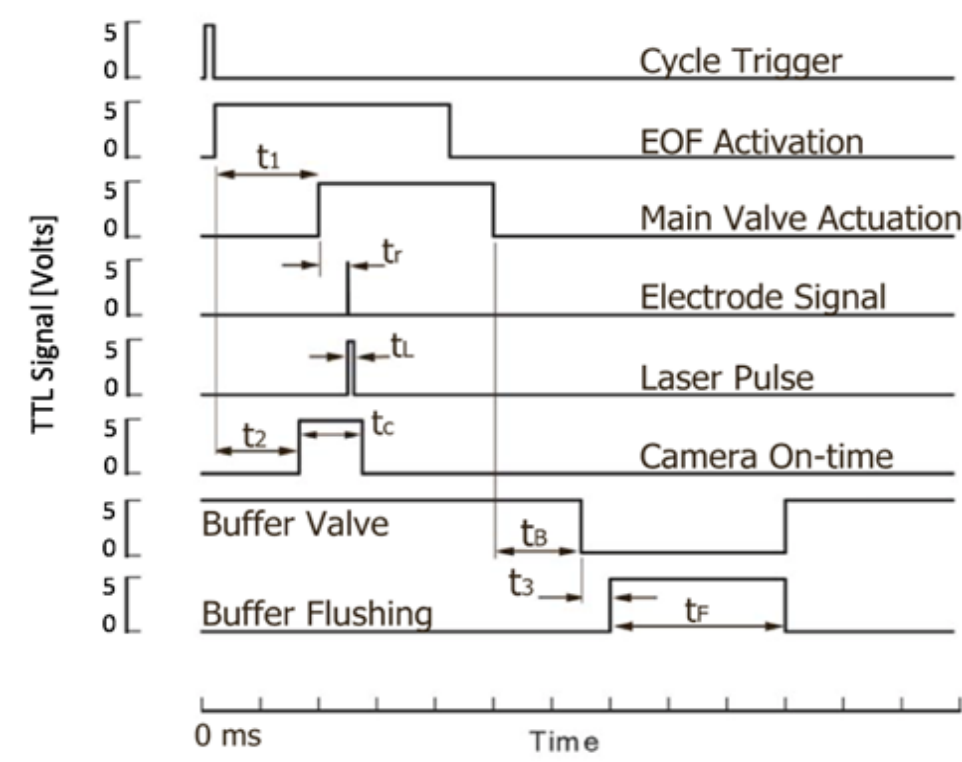

Figure 4: Triggering scheme for stroboscopic sub-millisecond fluorescence microscopy. All signal delays were triggered by the main cycle trigger, except for the laser pulse delay which was initiated by the electrical signal from the opening of the valve. 


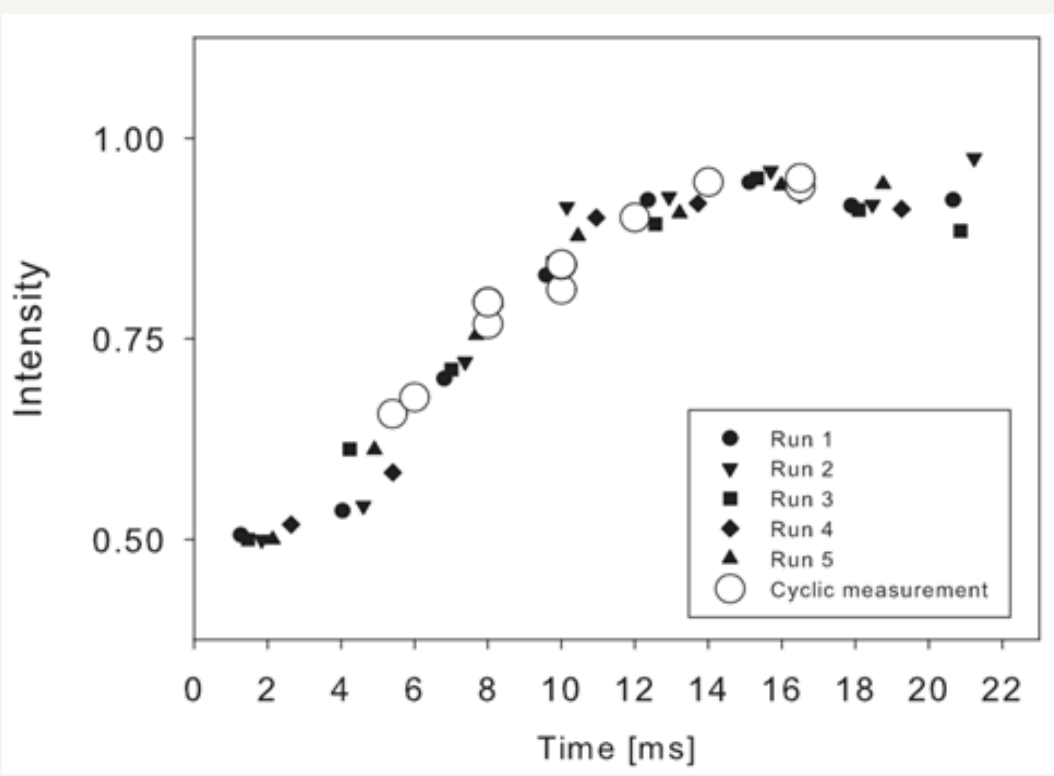

Figure 5: FITC fluorescence response to a $\mathrm{pH}$ change as a function of time after opening of the valve. The acidity of the buffer solution was changed from $\mathrm{pH} 7.6$ to $\mathrm{pH}$ 9.1. Results from individual runs with different delay constants $t_{\mathrm{R}}$ and cyclic measurements agree and allow to follow the buffer exchange, or more generally the response to the delivery of a reagent to surface immobilized probe molecules with sub-millisecond time resolution.

Figure 5 shows rapid buffer exchanges for $10 \mathrm{mM} \mathrm{NaBr}$ from pH 7.2 to $\mathrm{pH} 9.1$ where reagent delivery to the surface of the microfluidic channel were assayed by monitoring the $\mathrm{pH}$-dependent fluorescence of the FITC reporter molecules. The arm of the main channel containing the reporter molecules was first completely flushed with $\mathrm{NaBr}$ buffer at $\mathrm{pH} 7.2$, and a potential of $450 \mathrm{~V}$ was then applied to start the EOF. Using the triggering scheme detailed above and shown in (Figure 4), the valve was then opened to allow the NaBr buffer at pH 9.1 to flow through the area of interest. The fluorescence emission was acquired as a function of time and extracted from the images. In (Figure 5) we report the fluorescence as a measure of $\mathrm{pH}$ for those molecules located $37 \mu \mathrm{m}$ away from the valve edge and in the center of the main channel. Profiles for runs 1 through 5 were constructed by acquiring images at $2.3 \mathrm{~ms} /$ image with continuous laser excitation, while the cyclic measurement was done by acquiring images at $1.7 \mathrm{~ms} /$ image with a laser excitation pulse tL of $112 \mu$ s to increase the acquisition time resolution. Runs 1 through 5 were all time-adjusted for delays in the triggering and picture processing of the camera. The measured signal to noise ratio for the fluorescent measurements was approximately 150 and $10 \mathrm{~ms}$ was required to deliver the desired solution to the surfacetethered molecules of interest in between the electrodes.

\section{Conclusion}

We have developed a compact and reusable rapid buffer exchange microfluidic tool to analyze fast kinetics of reactions involving surface-immobilized molecules. We have demonstrated its utility by using pH-dependent FITC fluorescent molecules and shown that buffer exchange is feasible within $10 \mathrm{~ms}$. Key to obtaining such rapid reagent delivery to a surface is the use of EOF due to its plug flow profile and steep concentration gradients near the surface-tethered molecules of interest. Our data indicate that the actual delivery time of the reagents to the surface near the valve is limited primarily by the opening dynamics of the valve, and not longer by diffusion as seen in most PDF-based schemes. In addition, the presented microfluidic platform is highly stable and repeatable, and thus suitable for applications requiring the acquisition of high quality fluorescence images requiring discrete and homogeneous buffer exchange for an extended period of time. Using a triggering scheme with stroboscopic data acquisition, we followed the buffer exchange on a time scale of $10 \mathrm{~ms}$, which suggests that folding and unfolding kinetics of larger biomolecules can be followed with this type of device on the millisecond time scale using valve actuated EOF.

\section{Acknowledgement}

The author would like to thank Dr. Gerhard Blab for insights on methods, Dr. Jens-Christian Meiners for directing this project, Keith Shaw for the design and construction of the EOF power supply, Jason Kuo for sharing his method to bond PDMS chips for high pressure flows, and Brian Johnson, Mark Burns, and Robert Kennedy for access to the MEMS fabrication equipment. This work was in part funded by grants from the NIH (GM 65934-01) and NASA (NNC04AA21A and NAA64CD01G).

\section{References}

1. Shafer RH, Smirnov I (2000) Biological aspects of DNA/RNA quadruplexes. Biopolymers 56(3): 209-227.

2. Chen YF, Blab GA, Meiners IC (2009) Stretching submicron biomolecules with constant-force axial optical tweezers. Biophys J 96(11): 4701-4708.

3. Ding WX, Yin XM (2008) Sorting, recognition and activation of the misfolded protein degradation pathways through macroautophagy and the proteasome. Autophagy 4(2): 141-150. 
4. Soto C, Estrada LD (2008) Protein misfolding and neuro degeneration. Arch Neurol 65(2): 184-189.

5. Thirumalai D, Woodson SA (1996) Kinetics of Folding of Proteins and RNA. Acc Chem Res 29(9): 433-439.

6. Bokinsky G, Rueda D, Misra VK, Rhodes MM, Gordus A, et al. (2003) Single-molecule transition-state analysis of RNA folding. Proc Natl Acad Sci 100(16): 9302-9307.

7. Jarzynski C (2001) How does a system respond when driven away from thermal equilibrium? Proc Natl Acad Sci 98(7): 3636-3638.

8. Liphardt J, Onoa B, Smith SB, Tinoco I, Bustamante C (2001) Reversible unfolding of single RNA molecules by mechanical force. Science 292(5517): 733-737.

9. Phillips CM, Mizutani Y, Hochstrasser RM (1995) Ultrafast thermally induced unfolding of RNase A. Proc Natl Acad Sci 92(16): 7292-7296.

10. Lu HSM, Volk M, Kholodenko Y, Gooding E, Hochstrasser RM, DeGrado WF, et al. (1997) Aminothiotyrosine disulfide, an optical trigger for initiation of protein folding. J Am Chem Soc 119(31): 7173-7180.

11. Volk M, Kholodenko Y, Lu HSM, Gooding EA, DeGrado WF, Hochstrasser RM (1997) Peptide conformational dynamics and vibrational stark effects following photoinitiated disulfide cleavage. J Phys Chem B 101(42): 8607-8616.

12. Brewer LR, Corzett M, Balhorn R (1999) Protamine-induced condensation and decondensation of the same DNA molecule. Science 286(5437): 120-123.

13. Murphy RJ, Pristinski D, Migler K, Douglas JF, Prabhu VM, et al. (2010) Dynamic light scattering investigations of nanoparticle aggregation following a light-induced pH jump. J Chem Phys 132(19): 194903.

14. Abbruzzetti S, Carcelli M, Pelagatti P, Rogolino D, Viappiani C, et al. (2001) Photo induced alkaline $\mathrm{pH}$-jump on the nanosecond time scale. Chemical Physics Letters 344(3-4): 387-394.

15. Ma C, Steinmetz MG (2004) Substituent effects on competitive release of phenols and 1,3-rearrangement in $\alpha$-keto amide photochemistry. Org Lett 6(4): 629-632.
16. Knight JB, Vishwanath A, Brody JP, Austin RH (1998) Hydrodynamic focusing on a silicon chip: Mixing nanoliters in microseconds. Phys Rev Lett 80(17): 3863-3866.

17. Pollack L, Tate MW, Darnton NC, Knight JB, Gruner SM, et al. (1999) Compactness of the denatured state of a fast-folding protein measured by submillisecond small-angle x-ray scattering. Proc Natl Acad Sci 96(18): 10115-10117.

18. KCWLM R (2004) Review of Scientific Instruments 76: 014302.

19. Allen PB, Milne G, Doepker BR, Chiu DT (2010) Pressure-driven laminar flow switching for rapid exchange of solution environment around surface adhered biological particles. Lab Chip 10(6): 727-733

20. Hirono-Hara Y,Hiroyuki N, Takeuchi S (2009) Single-biomolecule observation with micro one-way valves for rapid buffer exchange. Journal of Applied Physics 105: 102016.

21. Erickson D, Liu X, Krull U, Li D (2004) Electrokinetically controlled DNA hybridization microfluidic chip enabling rapid target analysis. Anal Chem 76(24): 7269-7277.

22. Chen H, Acharya D, Gajraj A, Meiners J-C (2003) Robust interconnects and packaging for microfluidic elastomeric chips. Anal Chem 75(19): 5287-5291.

23. Chen H, Gu W, Cellar N, Kennedy R, Takayama S (2008) Electromechanical properties of pressure-actuated poly(dimethylsiloxane) microfluidic push-down valves. Anal Chem 80(15): 6110-6113.

24. (2008) H.B. Theoretical Microfluidics. Oxford master series in condensed matter physics, Oxford University Press, New York, USA.

25. Liu Y, Fanguy JC, Bledsoe JM, Henry CS (2000) Dynamic coating using polyelectrolyte multilayers for chemical control of electroosmotic flow in capillary electrophoresis microchips. Anal Chem 72(24): 5939-5944.

26. Kirby BJ, Hasselbrink EF (2004) Zeta potential of microfluidic substrates: 2. Data for polymers. Electrophoresis 25(2): 203-213.

27. Kirby BJ, Hasselbrink EF (2004) Zeta potential of microfluidic substrates: 1. Theory, experimental techniques, and effects on separations. Electrophoresis 25(2): 187-202. 